\title{
False contouring effect of the uterine wall during hysteroscopy a lesson from immediate hydrosonography
}

\author{
Yaron Hamani ${ }^{1}$, Ernst Voss $^{1}$, Asher Shushan ${ }^{1,2}$, Ronit Haimov-Kochman ${ }^{1,2^{*}}$ \\ ${ }^{1}$ Department of Obstetrics and Gynecology, Hadassah Medical Center, Mt. Scopus, Jerusalem \\ ${ }^{2}$ Women's Health Center, Maccabi Health Service, Modiin Ilit, Israel \\ Email: "kochman@hadassah.org.il
}

Received 31 March 2013; revised 1 May 2013; accepted 10 May 2013

Copyright @ 2013 Yaron Hamani et al. This is an open access article distributed under the Creative Commons Attribution License, which permits unrestricted use, distribution, and reproduction in any medium, provided the original work is properly cited.

\begin{abstract}
A 42 years old woman suffering from repeated events of intensive vaginal bleeding during the week off birth control pills was referred for diagnostic hysteroscopy. The patient underwent hysterohydrosonoscopy, a 3-step procedure, including a transvaginal ultrasound scan, a diagnostic hysteroscopy and immediate hydrosonography. A $3 \mathrm{~cm}$ intramural myoma was detected by the ultrasound scan without impinging onto the uterine cavity as visualized by diagnostic hysteroscopy. However, continuous hydrosonographic evaluation of the uterus, performed immediately afterwards, unveiled a fibroid with a significant intracavitary portion. A falsely normal cavity could be appreciated by high pressured hysteroscopy, while during the gradual fall of the intracavitary pressure a significant lesion could be revealed encroaching into the lumen. In the absence of an agreed distention pressure range during hysteroscopy, an immediate hydrosonography may serve as an auxiliary tool to follow the uterine wall contour as distention pressure declines during the eventual escape of the distention fluid from the lumen. Uterine wall contour is heavily dependent on the distension pressure and guidelines for intrcavitary pressure during hysteroscopy are evidently needed.
\end{abstract}

Keywords: Distension; Fibroid; Hydrosonography; Hysteroscopy; Pressure

\section{CASE REPORT}

A 42 years old woman was referred to the uterine imaging unit for repeated events of intensive vaginal bleeding during the week off birth control pills over three months

\footnotetext{
*Corresponding author.
}

prior to her referral. The patient underwent hysterohydrosonoscopy (HHSS).

HHSS has been described in detail previously [1]. It is a 3-step procedure including: 1) a transvaginal ultrasound scan (TVUS) in both sagittal and oblique transverse planes with a 6.5-MHz probe (MyLab, ESAOTA, Italy); 2) a diagnostic hysteroscopy (HS) for direct visualization of the uterine cavity by a rigid $30^{\circ}$ angle view, 5-mm hysteroscope (Karl Storz, Tuttlingen, Germany) with manually pressurized infusion of $100 \mathrm{~mm} \cdot \mathrm{Hg}$ of $0.9 \%$ saline solution to achieve sufficient distension of the cavity and visualization of the tubal ostia, and immediately after removing the hysteroscope; 3) hydrosonography (HSG) with the residual saline left inside the uterus [1]. HSG is undertaken without an additional insertion of a balloon catheter into the cervical canal. The procedure is done with neither local nor general anesthesia.

TUVS revealed a $3 \mathrm{~cm}$ intramural myoma in the posterior uterine wall (Figure 1a). The suspicion of a fibroid impinging onto the uterine cavity was deferred by diagnostic HS that revealed a normal uterine cavity (Figure 1b). However, continuous hydrosonographic evaluation of the uterus, performed immediately afterwards, during the gradual escape of the distention medium from the lumen, unveiled a fibroid with a significant intra-cavitary portion (Figures 1c-e). Measurements of the lesion were carried out during the state of hydrometra using two images with differential intracavitary volume (Figures 2a and $\mathbf{b})$. The level of uterine encroachment was calculated as $30 \%-50 \%$ of the entire lesion, and the myoma-serosal distance was found to be $6 \mathrm{~mm}$ (Figure 2a).

The patient tolerated the 3-step procedure of HHSS well. Based on the complete evaluation of the lumen with HHSS the patient was offered a hysteroscopic myomectomy to alleviate the bleeding. Levonorgestrel IUS was regarded as an unsuitable treatment modality due to 

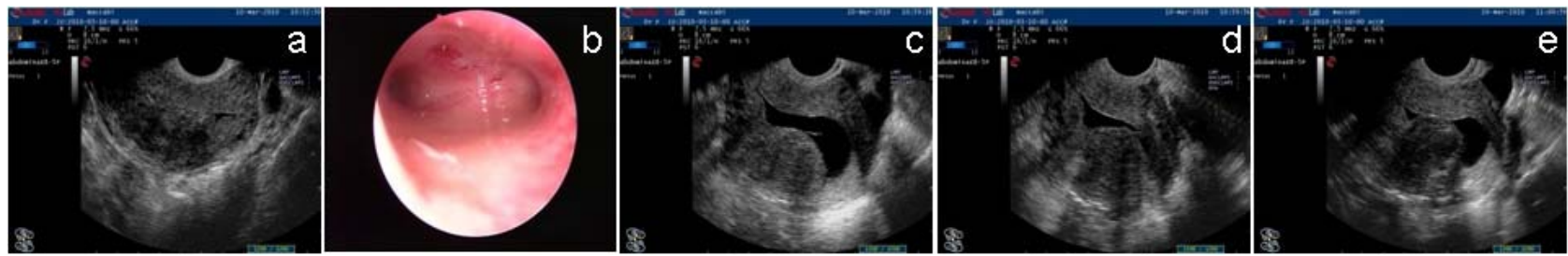

Figure 1. The 3-step procedure of hysterohydrosonoscopy (HHSS), disclosing a subserous myoma, which had been missed by hysteroscopy. a. Transvaginal ultrasound scan revealed a $3 \mathrm{~cm}$ intramural myoma in the posterior uterine wall; b. Diagnostic hysteroscopy with fully distended uterine cavity failed to disclose any abnormality; c-e. Continuous hydrosonographic evaluation of the uterus, performed immediately afterwards, during the gradual escape of the distention medium from the lumen, unveiled a fibroid with a significant intra-cavitary portion.
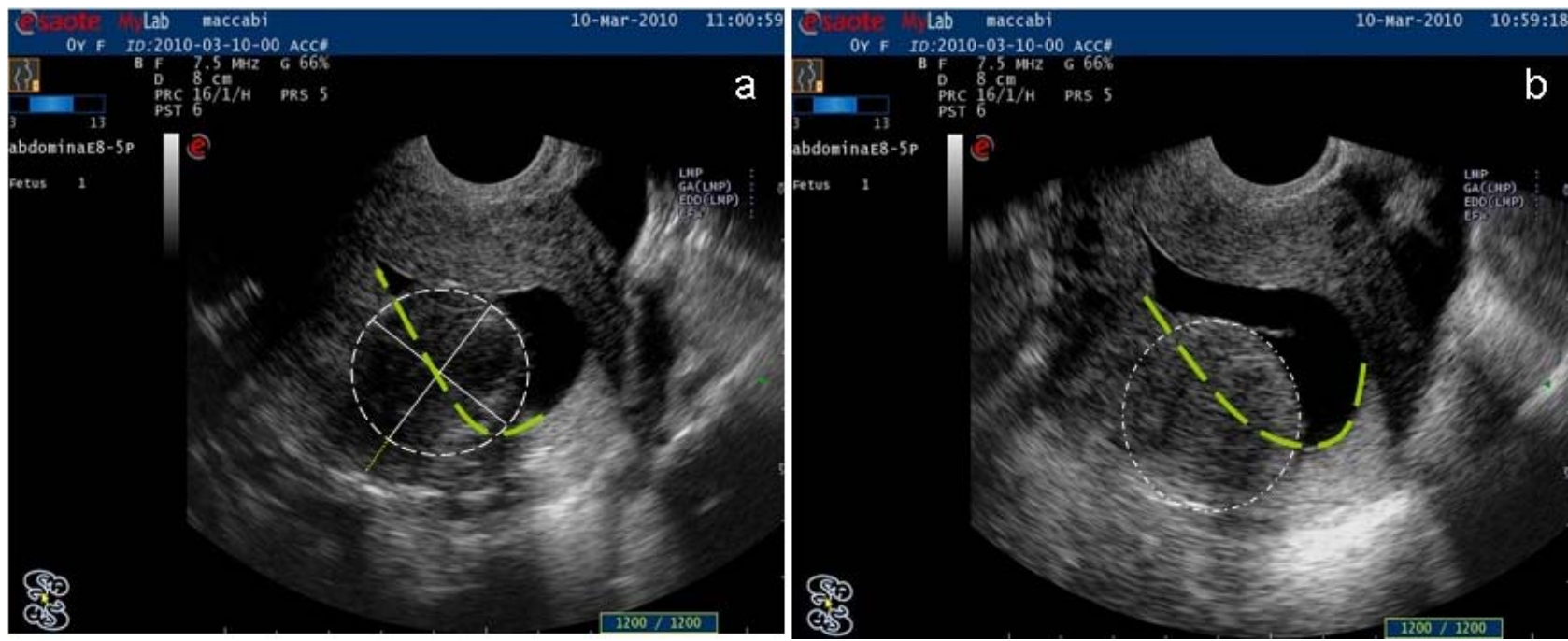

Figure 2. Measurements of the lesion during hysterography using two images with differential intracavitary volume (a, b). The level of uterine encroachment was calculated as $30 \%-50 \%$ of the entire lesion, and the myoma-serosal distance was found to be 6 mm.

the significant intracavity component of the lesion. Following our recommendation the patient underwent hysteroscopic myomectomy of a solitary $3 \mathrm{~cm}$ submucous fibroid. Diagnosis was confirmed by pathological evaluation. Symptomatic relief was reported shortly after the operation.

\section{DISCUSSION}

This case report highlights the value of HHSS in triaging women with fibroids for transcervical resection of fibroids (TCRM), either to treat menorrhagia or sub-fertility. HHSS is not widely used but simple and cheap to employ. There is a paucity of published reports supporting the technique. Centrally positioned small $(2-5 \mathrm{~cm})$ fibroids are commonly implicated as a cause of fertility and excessive menstrual bleeding. Conservative treatment options are limited; small fibroids may not be suitable for selective uterine artery embolisation, the levonorgestrel IUS and simple ablation may not be appropriate if there is a significant intracavity component. Exact positioning of a fibroid is enhanced by combining the 3 examination modalities described here: TVUS, HS and HSG.
We have previously reported the advantages of HHSS, a complementary HSG to diagnostic HS, especially in cases of abnormal uterine cavity [1]. HHSS simply uses residual saline in the uterus as an image enhancement rather than a dedicated infusion system. In a case series published recently [1] we have showed that in $92 \%$ of the procedures the remaining fluid did not escape before a full inspection of the cavity had been achieved. By HHSS the operator acquires of the ability to measure intracavitary lesions, and transform the non-quantitative hysteroscopic impression into a quantitative one. At this time we argue that complementary HSG could also fix the false impression of a normal uterine wall contour visualized by HS.

The view of the uterine cavity by HS is largely dependent on the intracavitary pressure of the distension medium. The usual intrauterine pressure required to visualize the endometrial cavity during HS when saline is the distending medium is $25-50 \mathrm{~mm} \cdot \mathrm{Hg}$, with pressures of 100 to $110 \mathrm{~mm} \cdot \mathrm{Hg}$ required to visualize the tubal ostia [2]. Infusion pressures during HS without anesthesia are usually set lower than while on general anesthesia because of the discomfort that higher pressures cause. 
In this case, we showed that a falsely normal cavity was noted by higher pressured HS, while during the gradual fall of intracavitary pressure a fibroid has been revealed with a significant portion encroaching into the lumen. This new datum is vital to the operator as the level of fibroid encroachment may determine the therapeutic options as well as the surgical approach for its excision [3-6].

Another common example of disagreement between endoscopists and US experts is the size of a uterine septum in relation to the cavity. In a fully distended highpressured HS the relative size of the septum may be underestimated, whereas in a low-pressured HS the septum may be overestimated. Here again, the relative length of the uterine septum usually dictates the need for septectomy in order to prevent unfavorable pregnancy outcome [7-10].

Today, pressure controlled continuous-flow system is widely accepted during operative HS, especially with low-viscosity fluids, to avoid distention media complications due to intravasation into the patient's vascular system [11]. The common practice among endoscopists is to recommend visualization of the uterine cavity with the lowest pressure needed. However, diagnostic HS is seldom carried out with a pressure monitoring, although the relation between intrauterine pressure and cavity volume has already been described by a typical hysteresis curve [12]. Pressure-controlled diagnostic HS was also shown recently to have the advantage of enabling close visualization of the surface appearance of the endometrium [13].

It may be argued that an effective survey of the uterine cavity should be performed using differential pressure; maximum pressure to ensure entire cavity viewed is followed by a lower pressure to allow for further assessment of the submucus component of a fibroid without the effect of a high hydrostatic pressure.

Guidelines for optimal distention pressure during HS are unavailable [14,15]. Similarly, guidelines have not been published for an acceptable intracavitary pressure in time of lesion measurements. Lack of agreed uterine pressure range for the description of intraluminal lesions contributes to inter-operator discrepancies and patient's confusion.

This pictorial study reinforces our advocacy that HSG may add important information immediately after HS that is pertinent to the patient's diagnosis and care. It also suggests that uterine wall contour is heavily dependent on the uterine distension pressure and guidelines for intrcavitary pressure during HS is evidently needed.

\section{REFERENCES}

[1] Haimov-Kochman, R., Pshitizky, M., Hamani, Y., Hur- witz, A. and Voss, E. (2010) HysterohydrosonoscopyAn integrated modality for uterine imaging. Gynecological Surgery, 7, 311-314. doi:10.1007/s10397-010-0576-1

[2] Baker, V.L. and Adamson, D.G. (1998) Minimum intrauterine pressure required for uterine distension. Journal of the American Association of Gynecologic Laparoscopists, 5, 51-53. doi:10.1016/S1074-3804(98)80011-X

[3] Lasmar, R.B., Barrozo, P.R., Dias, R. and Oliveira, M.A. (2005) Submucous myomas: A new presurgical classification to evaluate the viability of hysteroscopic surgical treatment-Preliminary report. Journal of Minimally Invasive Gynecology, 12, 308-311. doi:10.1016/j.jmig.2005.05.014

[4] Kolankaya, A. and Arici, A. (2006) Myomas and assisted reproductive technologies: When and how to act? Obstetrics \& Gynecology Clinics of North America, 33, 145-152. doi:10.1016/j.ogc.2005.12.008

[5] Bettocchi, S., Siristatidis, C., Pontrelli, G., Di Spiezio Sardo. A., Ceci, O., Nappi, L. and Selvaggi, L. (2008) The destiny of myomas: Should we treat small submucous myomas in women of reproductive age? Fertility and Sterility, 90, 905-910.

doi:10.1016/j.fertnstert.2007.09.015

[6] Sankaran, S. and Manyonda, I.T. (2008) Medical management of fibroids. Best Practice \& Research Clinical Obstetrics \& Gynaecology, 22, 655-676. doi:10.1016/j.bpobgyn.2008.03.001

[7] Fedele, L., Bianchi, S., Marchini, M., Mezzopane, R., Di Nola, G. and Tozzi, L. (1996) Residual uterine septum of less than $1 \mathrm{~cm}$ after hysteroscopic metroplasty does not impair reproductive outcome. Human Reproduction, 11, 727-729. doi:10.1093/oxfordjournals.humrep.a019242

[8] Rackow, B.W. and Arici, A. (2007) Reproductive performance of women with müllerian anomalies. Current Opinion in Obstetrics and Gynecology, 19, 229-237. doi:10.1097/GCO.0b013e32814b0649

[9] Ozgur, K., Isikoglu, M., Donmez, L. and Oehninger, S. (2007) Is hysteroscopic correction of an incomplete uterine septum justified prior to IVF? Reproductive BioMedicine Online, 14, 335-340. doi:10.1016/S1472-6483(10)60876-0

[10] Bozdag, G., Aksan, G., Esinler, I. and Yarali, H. (2008) What is the role of office hysteroscopy in women with failed IVF cycles? Reproductive BioMedicine Online, 17, 410-415. doi:10.1016/S1472-6483(10)60226-X

[11] Shirk, G.J. and Gimpelson, R.J. (1994) Control of intrauterine fluid pressure during operative hysteroscopy. Journal of the American Association of Gynecologic Laparoscopists, 1, 229-233. doi:10.1016/S1074-3804(05)81015-1

[12] Bajka, M., Weiss, S., Kunz, J., Fischer, H., Szkely, G. and Niederer, P. (2009) Pressure-dependent hydrometra dimensions in hysteroscopy. Surgical Endoscopy, 23, 2102-2109. doi:10.1007/s00464-008-0231-3

[13] Garry, R. (2010) Pressure-controlled hysteroscopy during menstruation. Journal of Minimally Invasive Gynecology, 17, 337-343. doi:10.1016/j.jmig.2010.01.009

[14] Siegler, A.M., Valle, R.F., Lindemann, H.S. and Men- 
caglia, L. (1990) Therapeutic hysteroscopy indication and techniques. CV Mosby, St. Louis.

[15] American College of Obstetricians and Gynecologists

\section{LIST OF ABBREVIATIONS}

HSG: hydrosonography

HHSS: hysterohydrosonoscopy

HS: hysteroscopy
(2005) ACOG technology assessment in obstetrics and gynecology, number 4, August 2005: Hysteroscopy. Obstetrics \& Gynecology, 106, 439-442.
IUS: intra uterine system

TCRM: transcervical resection of myomas

TVUS: transvaginal ultrasound scan 